Olivier MARTIN, L'Empire des chiffres. Une sociologie de la quantification

\title{
Marion Selz
}

\section{(2) OpenEdition}

1 Journals

\section{Édition électronique}

URL : https://journals.openedition.org/ress/7713

DOI : $10.4000 /$ ress. 7713

ISBN : $1663-4446$

ISSN : $1663-4446$

Éditeur

Librairie Droz

Édition imprimée

Date de publication : 6 décembre 2021

Pagination : 332-336

ISSN : 0048-8046

Référence électronique

Marion Selz, "Olivier MARTIN, L'Empire des chiffres. Une sociologie de la quantification », Revue européenne des sciences sociales [En ligne], 59-2 | 2021, mis en ligne le 01 décembre 2021, consulté le 09 décembre 2021. URL : http://journals.openedition.org/ress/7713 ; DOI : https://doi.org/10.4000/ ress. 7713

Ce document a été généré automatiquement le 9 décembre 2021.

(ㄷ) Librairie Droz 


\title{
Olivier MARTIN, L'Empire des chiffres. Une sociologie de la quantification
}

\author{
Marion Selz
}

\section{RÉFÉRENCE}

Olivier MARTIN, 2020, L'Empire des chiffres. Une sociologie de la quantification, Paris, Armand Colin, 298 p.

1 Si le livre d'olivier Martin évoque bien, au fil des pages, l'envahissement par les chiffres de tous les espaces de notre société comme le titre nous y fait penser, il parle aussi de beaucoup d'autres choses : depuis leur naissance d'aussi loin que l'on peut les repérer jusqu'à leur utilisation contemporaine, il décortique leur fabrication, montre comment s'élabore la quantification. Il s'agit en fait de l'histoire de leur empire sous de multiples aspects.

2 Tout d'abord il est peut-être nécessaire de préciser, comme l'auteur le fait dans l'introduction de son ouvrage, au cours d'une stimulante leçon de terminologie, explicitant notamment la distinction entre l'usage de " chiffre » et celui de "nombre ", ce que les chiffres désignent ici : "c'est le résultat d'un processus de quantification, exprimant donc une quantité ou une appréciation quantitative» (p. 7). Cette histoire est décrite, dans la première partie de l'ouvrage, par les nécessités auxquelles les chiffres répondent et par les pratiques et techniques de leur élaboration, à travers plusieurs exemples ; la seconde partie spécifie les raisons qui les justifient et les effets qu'ils produisent dans la société.

3 Le livre nous plonge d'emblée (chapitre 1) dans la préhistoire au cours de laquelle on trouve déjà des traces, préexistant même à l'écriture, de l'activité de compter et de dénombrer à des fins organisationnelles, administratives et fiscales : c'est une nécessité pour pouvoir partager les terres, délimiter des domaines privés ou publics, enregistrer et authentifier des parcelles dans des registres de cadastres. Peu à peu l'usage des chiffres s'installe dans de nombreux domaines, certains d'entre eux faisant l'objet des 
chapitres suivants. L'habitude de plus en plus courante d'échanges commerciaux donne lieu à l'invention de mesures des marchandises (chapitre 2). La mesure du temps, essentielle pour contrôler le travail des employés, et l'évolution des divers instruments de plus en plus sophistiqués qui la permettent, sont décrites dans le chapitre 3. Le chapitre 4 montre que suite à la naissance de la statistique à la fin du XVIII siècle, l'omniprésence des données statistiques règne dans notre monde, que ce soit à des fins de pouvoir ou de connaissance. Le chapitre 5 explicite l'interaction des sciences avec la quantification, le XIX ${ }^{e}$ siècle étant déterminant dans le passage des mesures pratiques aux mesures scientifiques dans divers domaines de la physique mais aussi en psychologie et en sociologie. Dans le chapitre 6, Olivier Martin appréhende la question des échelles (p. 136: nominales, ordinales, d'intervalle ou de rapport) inhérente aux mesures, et celle des opérations pouvant s'y effectuer selon leur type : il évoque la nonproportionnalité et non-additivité de certaines d'entre-elles, comme par exemple la mesure de la température. Le chapitre 7 clôt la première partie en complétant l'avalanche des chiffres qui nous entoure, déjà abordée au chapitre 4 , mais en insistant plus particulièrement sur les mesures de la performance, de l'efficacité, de la qualité, que ce soit d'individus, ou d'entités (établissements, institutions, etc.).

4 Les trois chapitres constituant la seconde partie sont en quelque sorte une synthèse issue de la première et en donne une vue transversale, occasionnant des répétitions sans nuire à la lecture. Le chapitre 8 récapitule la quantification comme fait social, entièrement construite selon des conventions. Le chapitre 9 indique les raisons qui la justifient: l'aide à la décision, la recherche d'objectivité et de scientificité, une meilleure connaissance de phénomènes. Le chapitre 10 précise ses effets, dont certains peuvent être pervers.

$5 \quad$ L'aspect le plus marquant de ce livre tient au fait qu'il concerne tous les chiffres (les numéros ou codes qui sont des labels n'exprimant pas des quantités, ainsi que ceux de la monnaie, l'argent et la comptabilité, quoique faisant partie du projet global de l'ouvrage, n'ont cependant pas été retenus), faisant ainsi apparaître leurs points communs. Abolissant la distinction entre mesure et quantification (p. 184), l'auteur développe abondamment le fait que la quantification est, depuis toujours, non pas naturelle, mais bien un fait social (p. 177), une construction au sein de laquelle il pointe le rôle du pouvoir (p. 229). Il montre ce faisant comment la science a utilisé les chiffres pour se construire avant que ceux-ci en soient, de plus en plus souvent, les produits.

6 Il s'agit bien de tous les chiffres (p.8): des mesures individuelles aux mesures collectives, des indicateurs socio-économiques ou psychologiques aux résultats d'analyses biologiques en passant par les horaires de trains et les mesures météorologiques ou astrologiques, s'appliquant à des objets, à des individus ou à des événements, quelle que soit leur provenance : des sciences de la nature, de conventions culturelles, d'activités politiques ou marchandes. À rebours de tous les travaux existants qui établissent une division nette entre mesure et quantification, Olivier Martin efface cette séparation : il s'oppose notamment à la posture d'Alain Desrosières (2014, Prouver et Gouverner. Une analyse politique des statistiques publiques, Paris, La Découverte), à bien juste titre souvent cité dans cet ouvrage, qui distingue explicitement ces deux notions. Ce dernier considère en effet que la mesure se réfère à quelque chose de naturellement chiffrable, selon une métrologie réaliste, alors que la quantification suppose d'emblée l'élaboration de conventions préalables, et est, de ce fait, relativiste. 
7 Selon Olivier Martin, aucun chiffre n'est naturel. Quand bien même certains sont construits à partir de propriétés physiques de la terre ou de l'homme (p. 34), comme le pouce, le pied, l'empan, la coudée, le pas ou encore la capacité de travail, la journée, l'hommée (mesure de terre que l'homme peut cultiver, faucher, labourer, en une journée), ils résultent toujours d'un processus : ils ne sont pas «donnés» naturellement, mais fabriqués par l'homme. Plusieurs exemples illustrent ce point de vue: celui de la construction du système métrique (p.45), de la mesure du temps (p. 53), de la température (p.133), de l'intelligence (p.142), du chômage (p. 97). Leur histoire est l'occasion de riches développements très intéressants. Dans tous ces cas, le choix d'une unité de mesure est une véritable élaboration, découlant de conventions collectives, fruits de concertations parfois longues entre différentes parties. Il est une partie intrinsèque du processus de quantification, considéré ici comme le point commun à tous les chiffres.

8 La quantification est un fait social total. C'est une activité qui date de la préhistoire : arpenteur est un des plus vieux métiers du monde, et on trouve des traces de recensement de la population plusieurs siècles avant le début de notre ère dans plusieurs parties du monde. Dès leur naissance, l'utilisation de chiffres répond à une nécessité sociale, qu'il s'agisse de coordonner des activités, de se comprendre, d'organiser la vie sociale, d'échanger des biens, de délimiter des terres, de prélever des taxes, etc. (p. 27-28).

9 Les pratiques chiffrées sont d'abord locales (l'arpent variait selon les régions) et artisanales. Puis le développement et l'extension des échanges commerciaux à des territoires de plus en plus larges, la volonté des pouvoirs de contrôler ces échanges et de prélever des impôts, celle des individus de pouvoir comparer pour dénoncer les injustices ont, au fil d'un temps long, forgé «l'abstraction, l'universalisation, l'uniformisation" des chiffres, non sans surmonter réticences, conflits et multiples difficultés tant matérielles que philosophiques. Ces trois notions, convoquées à plusieurs reprises dans l'ouvrage, sont concomitantes au sein d'un même processus de quantification dont elles sont les constituants essentiels. Olivier Martin précise la façon dont chacune de ces notions opère effectivement, au cours de ce processus (p. 41-43).

10 L'abstraction consiste à ne garder des choses quantifiées qu'une seule et unique caractéristique les décrivant au détriment des autres, retenue selon la circonstance. Ainsi les divers récipients spécifiques à chaque marchandise ont été abandonnés au profit d'un seul trait qui leur est commun : leur poids. De même on peut ne retenir de certaines choses que la longueur, la même unité permettant de mesurer aussi bien un territoire qu'une distance ou la taille d'un objet. Au cours de « la mise en statistique de la société " actuelle (chapitre 4), de nombreux indicateurs sont élaborés en vue de décrire des aspects de la réalité sociale : le PIB, le QI, le taux de chômage, etc., pour n'en citer que quelques-uns, détaillés dans le livre. Ils sont toujours le fruit de conventions, nationales ou internationales. Chacun d'entre eux n'offre qu'un point de vue sur le concept qu'il est censé représenter : il en est bien une abstraction, et il en devient aussi, du fait de l'utilisation qui en sera faite, une réification.

11 L'universalisation désigne le fait qu'un même système de mesure permet d'appréhender un ensemble varié de choses. Elle est produite par l'abstraction en rendant possible l'utilisation par tout le monde des indicateurs construits conventionnellement. L'uniformisation consiste en l'adoption géographiquement de plus en plus vaste des mêmes indicateurs : au sein de tout un pays, puis d'un ensemble 
de pays. La quantification est d'autant plus un fait social qu'à partir du XXe siècle elle envahit la société, tant parce que tout est quantifié que parce qu'elle agit sur la société. En réifiant une réalité complexe, elle la simplifie et en devient la valeur vraie, la seule sur laquelle il faudrait agir pour la faire progresser. Elle est un instrument de gouvernement, mais aussi de connaissance de la société par le peuple, permettant d'identifier ses maux pour mieux les gérer, voire les résoudre. Sans pour autant qu'il soit question de nier ses apports, de nombreux exemples de ses effets pervers sont décrits : on peut agir pour améliorer une note sans faire progresser le problème de fond (p.157), l'évaluation permanente des travailleurs provoque un stress chronique en exacerbant leur mise en concurrence ( $\mathrm{p}$. 161), la publication du palmarès des lycées favorise la ségrégation sociale, la publication de sondages influence le vote des électeurs, etc. (p. 167).

De tout temps, les chiffres ont été façonnés par des pouvoirs (p. 195), qu'ils soient religieux, corporatistes, économiques ou politiques. Ils jouent un rôle essentiel, voire déterminant, dans les définitions et usages des systèmes de mesure en décidant des conventions, choisissant les indicateurs parfois utilisés dans les règlements et lois. La quantification, à travers la statistique née à la fin du XVIII ${ }^{e}$ siècle, devient peu à peu un outil incontournable des États modernes, que leurs dirigeants utilisent comme instrument de gouvernement au service de prises de décisions, d'arbitrages, d'élaboration et d'évaluation de politiques publiques.

Olivier Martin s'intéresse enfin aux interactions de la quantification avec la science (chapitre 5). Il insiste sur le fait que, contrairement à ce que l'on pourrait penser actuellement, la science n'est pas à l'origine de la quantification. Ce n'est que peu à peu, à partir du XVIII et surtout du XIX ${ }^{e}$ siècle, que les pratiques et la science se rencontrent. La réforme du système des poids et mesures lors de la Révolution française, réforme non seulement technique et sociale, mais aussi scientifique, en est une bonne illustration. Elle est suivie, en 1875, par la création du Bureau international des Poids et Mesures, instance à la fois savante et administrative. La quantification devient dès lors, progressivement, un acte de connaissance. Alors que diverses mesures ont permis à la science de se déployer, c'est ensuite cette science et les instruments qu'elle contribue à fabriquer qui permettent de connaitre de nombreuses mesures. L'exactitude et la précision se substituent à l'à-peu-près. Les sciences deviennent « exactes». En effet, d'abord approximatives, les mesures sont peu à peu l'objet d'exigence de précision toujours plus grande et de comparaisons, aboutissant à l'invention d'appareils scientifiques qui permettent ensuite d'accéder à de nouvelles connaissances de notre monde. La mesure du temps, depuis le rythme des journées par les lever et coucher du soleil, jusqu'à l'établissement d'un temps universel et la réalisation d'outils scientifiques au service des physiciens et astronomes, en passant par les cadrans solaires, les cloches, les clepsydres, les sabliers, les calendriers, les horloges, les montres, etc., en est une convaincante illustration qui fait l'objet de tout un chapitre.

14 De nombreux phénomènes, comme le magnétisme, l'électricité, la chimie, de même que l'astronomie, la mécanique, la cinématique l'optique ont été identifiés, circonscrits et étudiés par les savants de façon essentiellement qualitative avant d'être approfondis grâce à des mesures expérimentales, contredisant l'évidence, d'après l'auteur largement répandue aujourd'hui, selon laquelle la quantification serait indispensable à la connaissance scientifique. La « quantophrénie » ambiante dans les sciences humaines 
et sociales par l'intermédiaire des statistiques et des probabilités, à des fins de légitimation et de scientificité, donne une apparence scientifique à la description de faits sociaux. Elle fait croire à l'objectivité de leur analyse alors que la bonne interprétation des chiffres exige une connaissance pointue de tout le processus de leur construction, laquelle n'est pas toujours acquise ou appliquée correctement par ceux qui y recourent, qu'ils soient politiques, décideurs, ou journalistes.

L'ouvrage fait montre d'une grande érudition. Il contient beaucoup d'informations très intéressantes et détaillées sur la mise en place progressive de la quantification dans nombre de domaines et circonstances, ainsi que des éléments de réflexion sur la théorisation de la notion de mesure. Il contient une bibliographie impressionnante tant par sa richesse que par la diversité des disciplines et des auteurs cités : sociologues, démographes, historiens, philosophes, statisticiens sont ainsi mis à contribution. À l'heure où les chiffres s'infiltrent dans tous les domaines de nos vies, cette synthèse générale peut conduire ses lecteurs à en être de meilleurs interprètes.

\section{AUTEURS}

\section{MARION SELZ}

CNRS, Paris - Centre Maurice Halbwachs 